\title{
Modification of Loudness Discomfort Level: Evidence for Adaptive Chronic Auditory Gain and Its Clinical Relevance
}

\author{
Craig Formby, Ph.D., ${ }^{1}$ and Susan Gold, M.A.1
}

The Publisher regrets errors in the article that originally appeared in Seminars in Hearing, volume 23, number 1, 2002. The corrected text is given below.

\section{ABSTRACT}

A vexing problem in audiology has been the modification of sound tolerance. Sound intolerance and restricted dynamic ranges are common conditions that audiologists encounter daily in the hearingimpaired population, especially in the fitting of hearing aids. To date, no clinical protocol has proven to be successful for modifying sound tolerance among the hearing-impaired population. This report describes the use of low-level, broadband sound in a habituation-based treatment protocol termed Tinnitus Retraining Therapy (TRT). Over the past decade, TRT has become increasingly popular as an intervention for severe tinnitus and hyperacusis. One of the primary treatment effects from TRT is that over the course of the intervention the patient's loudness discomfort levels (LDLs) routinely shift to higher levels. Ostensibly, the resulting higher LDLs reflect treatment effects associated with the resetting of a plastic and adaptive auditory gain process that somehow regulates the suprathreshold sensitivity of the auditory system in response to chronic changes in the sound input from the auditory periphery to the central auditory pathways. Tinnitus patients with significant hearing losses and sound tolerance problems respond successfully to TRT and, as a consequence, their LDLs are usually elevated (improved) and, concomitantly, their dynamic ranges are expanded. Many of these patients, who before the TRT intervention could not tolerate amplified sound, then have been able to make a comfortable transition into appropriate amplification after treatment. In principle, TRT would seem to offer a viable intervention strategy for modifying sound tolerance in the general hearing-impaired population. If so, then clinical applications of TRT principles may extend well beyond the treatment of tinnitus and hyperacusis.

\footnotetext{
New Frontiers in the Amelioration of Hearing Loss: Part I-Aural Rehabilitation and Sensory Aids; Editor in Chief, Catherine V. Palmer, Ph.D.; Guest Editor, Ann Geers, Ph.D. Seminars in Hearing, volume 23, number 1, 2002. Address for correspondence and reprint requests: Craig Formby, Ph.D., Division of Otolaryngology-HNS 16 South Eutaw, Suite 500, The University of Maryland School of Medicine, Baltimore, MD 21201. E-mail: cformby@smail.umaryland.edu. ${ }^{1}$ Division of Otolaryngology-HNS and The University of Maryland Tinnitus and Hyperacusis Center, The University of Maryland School of Medicine, Baltimore, Maryland. Copyright (C) 2002 by Thieme Medical Publishers, Inc., 333 Seventh Avenue, New York, NY 10001, USA. Tel: +1(212) 584-4662. 00734-0451,p;2002,23,02,179,180,ftx,en;sih00210x.
} 
In the abstract and body of the original article, the terminology "threshold" was inadvertently used in association with loudness discomfort level (LDL). The abstract has been reprinted here to correct this mistake, which is an erratum also applicable to the corresponding terminology in the body of the report.

Page $31,1^{\text {st }}$ column, $1^{\text {st }}$ paragraph, last three sentences: Consider, for example, the discussion by Allen and his colleagues of the results for their Loudness Growth in $1 / 2$ Octave Bands (LGOB) protocol. ${ }^{83}$ These investigators described findings for a pair of audiometrically normal subjects who appeared to show a form of loudness recruitment based upon their abnormally reduced response patterns for LOUD and TOO LOUD sound levels. ${ }^{83(\mathrm{p} \text { 752) The }}$ anomaly they were describing was likely hyperacusis rather than loudness recruitment.

\section{REFERENCE}

83. Allen JB, Hall JL, Jeng PS. Loudness growth in $1 / 2$ octave bands (LGOB): a procedure for the assessment of loudness. J Acoust Soc Am 1990;88: $745-753$ 
